\title{
REFLEXIVITY OF CYCLIC BANACH SPACES
}

\author{
L. TZAFRIRI ${ }^{1}$
}

In a Banach space $\mathfrak{X}$ with an unconditional basis $\left\{e_{n}\right\}$ the projections $E(\sigma) ; \sigma \subset N=\{1,2,3, \cdots, n, \cdots\}$ defined by $E(\sigma)\left(\sum_{n=1}^{\infty} \alpha_{n} e_{n}\right)$ $=\sum_{n \in \sigma} \alpha_{n} e_{n} ; \sum_{n=1}^{\infty} \alpha_{n} e_{n} \in \mathfrak{X}$ form a $\sigma$-complete atomic Boolean algebra of projections $\varepsilon$ for which there exists a vector $x_{0} \in \mathfrak{X}$ (for instance, $\left.x_{0}=\sum_{n=1}^{\infty} e_{n} / 2^{n}\left\|e_{n}\right\|\right)$ such that $\mathfrak{X}=\operatorname{clm}\left\{E x_{0} \mid E \in \mathcal{E}\right\}$. Viewed from this point, the Banach spaces having unconditional basis form a subclass of the family of cyclic spaces $\mathfrak{X}=\operatorname{clm}\left\{P x_{0} \mid P \in \Theta\right\}$ for some $x_{0} \in \mathfrak{X}$ and a $\sigma$-complete (not-necessarily atomic) Boolean algebra of projections $B$ on $\mathfrak{X}$. Cyclic spaces have been introduced by $W$. G. Bade [1], [2] in connection with the multiplicity theory for spectral operators on Banach spaces. A typical example is $L_{1}(0,1)$, the space of all integrable functions on $[0,1]$, which has no unconditional basis (cf. A. Pełczyński [13, Proposition 9]) but is a cyclic space with respect to the Boolean algebra of projections consisting of "multiplications" by characteristic functions.

W. G. Bade suggested recently in a discussion that it might follow from the theory of normed lattices that a cyclic space is reflexive provided its second conjugate is separable. Using a theorem of T. Ogasawara [12] on normed Riesz spaces we shall be able to prove in the present note that reflexivity of a cyclic space $\mathfrak{X}$ is insured by the condition (weaker than separability of the second conjugate) that neither $l_{1}$ nor $c_{0}$ would be isomorphic to a subspace of $\mathfrak{X}$. This result generalizes a well-known characterization of reflexivity for spaces with unconditional bases given by R. C. James [5].

Other properties of Banach spaces in connection with Boolean algebras of projections have been described recently in [6], [7], [10], [14].

1. Preliminaries. In this section we shall summarize briefly some notion and results needed in the sequel. A Boolean algebra of projections $B$ is called complete (cf. W. G. Bade [1]) if for every family $P_{\alpha} \in \mathbb{B}$ the projections $\vee P_{\alpha}$ and $\Lambda P_{\alpha}$ exist in $B$ and satisfy

$$
\left(\bigvee P_{\alpha}\right) \mathfrak{X}=\operatorname{clm}\left\{P_{\alpha} \mathfrak{X}\right\} ; \quad\left(\Lambda P_{\alpha}\right) \mathfrak{X}=\bigcap\left(P_{\alpha} \mathfrak{X}\right) .
$$

If $B$ is complete then there is a uniform bound $M$ for the norms of the projections $P \in B$ (cf. W. G. Bade [1, Theorem 2.2]). Regarding $B$ as

Received by the editors May 20, 1968 and, in revised form, February 5, 1969.

1 Supported by National Science Foundation Grant GP-7475. 
a spectral measure $P(\cdot)$ on the Borel sets $\Sigma$ of its Stone space $\Omega$, it follows from N. Dunford [3] that for every bounded Borel function $f$, the integral $S(f)=\int_{\Omega} f(\omega) P(d \omega)$ exists in the uniform operator topology and satisfies:

$$
\|S(f)\| \leqq 4 M \sup _{\omega \in \Omega}|f(\omega)| \text {. }
$$

If $f$ is not bounded and $e_{m}=\{\omega|\omega \in \Omega| f,(\omega) \mid \leqq m\}, m=1,2, \cdots$, the operator $S(f)$ is unbounded having the domain

$$
D(S(f))=\left\{x \mid x \in \mathfrak{X}, \lim _{m \rightarrow \infty} \int_{e_{m}} f(\omega) P(d \omega) x \text { exists }\right\} .
$$

In presenting definitions and results concerning normed lattices we will make use of the terminology and references from W. A. J. Luxemburg and A. C. Zaanen [8, Notes VI and XIII]. Accordingly, a real Banach space $L$ is called a complete Riesz normed space if it is partially ordered by $\leqq$ such that:

(i) $u \leqq v$ implies $u+w \leqq v+w$ for every $u, v, w \in L$.

(ii) $u \geqq 0$ implies $\alpha u \geqq 0$ for every $\alpha \geqq 0$.

(iii) For every pair $u, v \in L$, the least upper bound $\sup (u, v)$ and the greatest lower bound $\inf (f, g)$ exist in $L$.

(iv) The norm satisfies $\|u\| \leqq\|v\|$ if $|u| \leqq|v|$ (where $|u|=$ $\sup (u,-u))$.

A Riesz space $L$ is said to be $\sigma$-Dedekind complete if every sequence in $L$ which is bounded from above has a least upper bound. The notations $u_{\tau} \downarrow 0$ for a net $\left\{u_{\tau}\right\} \subset L$ means $\left\{u_{\tau}\right\}$ is a decreasing net whose greatest lower bound is zero.

The following theorem due to T. Ogasawara [12, Chapter V, $\$ 4$, Theorem 1] is stated here in the form found in W. A. J. Luxemburg and A. C. Zaanen [8, Note XIII, Theorem 40.1].

Theorem A. A complete Riesz normed space $L$ is reflexive if and only if the following three conditions are satisfied:

(a) $u_{\tau} \downarrow 0$ implies $\left\|u_{\tau}\right\| \rightarrow 0$ for every net $\left\{u_{\tau}\right\} \subset L$.

(b) $\phi_{\tau} \downarrow 0$ implies $\left\|\phi_{\tau}\right\| \rightarrow 0$ for every net $\left\{\phi_{\tau}\right\} \subset L^{*}$ (L* is the conjugate of $L$ ).

(c) $u_{\tau} \geqq 0$ and sup $\left\|u_{\tau}\right\|<+\infty$ implies sup $_{\tau} u_{\tau} \in L$ for every increasing net $\left\{u_{\tau}\right\} \subset L$.

2. Reflexivity of $\mathfrak{M}\left(x_{0}\right)$. Throughout this section $B$ will denote a complete Boolean algebra of projections on the Banach space $\mathfrak{X}$ for which there exists $x_{0} \in \mathfrak{X}$ such that

$$
\mathfrak{X}=\mathfrak{M}\left(x_{0}\right)=\operatorname{clm}\left\{P x_{0} \mid P \in B\right\} .
$$


The uniform bound for the norm of the projections $P \in B$ will be denoted by $M$. According to W. G. Bade [2, Theorem 4.5],

$$
\mathfrak{X}=\left\{S(f) x_{0} \mid x_{0} \in D(S(f))\right\} \text {. }
$$

LEMma 1. For each $x \in \mathfrak{X}$, define $|x|$ by $|x|=\sup \|S(\phi) x\|$ where the supremum is taken over all Borel functions $\phi$ for which $|\phi(\omega)| \leqq 1 ; \omega \in \Omega$.

Then $|\cdot|$ is a norm on $\mathfrak{X}$ equivalent to the original norm $\|\cdot\|$ and such that:

(a) $\|x\| \leqq|x| \leqq 4 M\|x\| ; x \in \mathfrak{X}$.

(b) If $S\left(f_{1}\right) x_{0} \in \mathfrak{X}$ and $f_{1}(\omega) \geqq f_{2}(\omega) \geqq 0 ; \omega \in \Omega$ for some Borel function $f_{2}$ then $S\left(f_{2}\right) x_{0} \in \mathfrak{X}$ and $\left|S\left(f_{1}\right) x_{0}\right| \geqq\left|S\left(f_{2}\right) x_{0}\right|$.

(c) $|P| \leqq 1 ; P \in B$.

(d) $\left|S(f) x_{0}\right|=\left|S(|f|) x_{0}\right| ; S(f) x_{0} \in \mathfrak{X}$.

The proof follows immediately from the definition of $|x|$ and properties of operators $S(f)$, and we omit it.

Denote $\mathfrak{X}^{(r)}=\left\{x \in \mathfrak{X}\left|x=S(f) x_{0}\right| f\right.$ real $\}$. Obviously $\mathfrak{X}^{(r)}$ is a real Banach space which can be ordered by setting $S\left(f_{1}\right) x_{0} \preceq S\left(f_{2}\right) x_{0}$ whenever $f_{1}(\omega) \leqq f_{2}(\omega)$ a.e. in $\Omega$. Let us remark that $\mathfrak{X}^{(r)}$ can be considered as the "real part" of $\mathfrak{X}$. In view of Lemma 1, part (b) and the Lebesgue Dominated Convergence Theorem for vector measures (cf. [4, IV-1010]) we have the following lemma.

Lemma 2. $\left\{\mathfrak{X}^{(r)}, \preceq\right\}$ is a complete Riesz normed space. Moreover, it is $\sigma$-Dedekind complete.

Now, denote as usual by $c_{0}$ the space of sequences convergent to zero and by $l_{1}$ the space of sequences whose series are absolutely convergent.

Lemma 3. If no subspace of $\mathfrak{X}^{(r)}$ is isomorphic to $c_{0}$ then for every increasing sequence $0 \preceq S\left(f_{1}\right) x_{0} \preceq S\left(f_{2}\right) x_{0} \preceq \cdots$ with $\sup _{n}\left|S\left(f_{n}\right) x_{0}\right|<+\infty$ we have $x_{0} \in D\left(S\left(\sup _{n} f_{n}\right)\right)$, i.e. $S\left(\sup _{n} f_{n}\right) x_{0} \in \mathfrak{X}^{(r)}$.

Proof. Assume there exists in $\mathfrak{X}^{(r)}$ an increasing sequence $0 \preceq S\left(g_{1}\right) x_{0} \preceq S\left(g_{2}\right) x_{0} \preceq \cdots$ with $\left|S\left(g_{n}\right) x_{0}\right| \leqq K, n=1,2, \cdots$ such that $g(\omega)=\sup _{n} g_{n}(\omega)$ is not integrable with respect to the vector measure $P(\cdot) x_{0}$. According to W. G. Bade [2, Theorem 4.3], there exists a functional $x_{0}^{*} \in\left(\mathfrak{X}^{(r)}\right)^{*}$ such that $\mu(\cdot)=x_{0}^{*} P(\cdot) x_{0}$ is a positive measure equivalent to the vector measure $P(\cdot) x_{0}$. Since

$$
\int_{\Omega} g_{n}(\omega) \mu(d \omega) \leqq K\left\|x_{0}^{*}\right\|, \quad u=1,2, \cdots,
$$

by Fatou's Lemma (cf. [4, III-6-19]), $g$ is integrable with respect to 
$\mu$ and therefore it is finite a.e. in $\Omega$. Consequently $\Omega=\bigcup_{m=1}^{\infty} \delta_{m}$ where $\delta_{m}=\{\omega \mid \omega \in \Omega, m-1 \leqq g(\omega)<m\}$. By a theorem of Lusin (cf. [4, III6-3]) we can assume with no loss of generality that $\left\{g_{n}\right\}$ converges $\mu$-uniformly to $g$, i.e., there exists a sequence of disjoint Borel sets $\left\{\sigma_{p}\right\}$ such that $\Omega=\bigcup_{p=1}^{\infty} \sigma_{p}$ and $\left\{g_{n}\right\}$ converges uniformly to $g$ on every set $\sigma_{p}, p=1,2, \cdots$. The subsets $\delta_{m} \cap \sigma_{p}, m, p=1,2, \cdots$ form a sequence of disjoint subsets of $\Omega$ which will be denoted by $\left\{\eta_{k}\right\}$. Obviously, $\Omega=\bigcup_{k=1}^{\infty} \eta_{k}$.

If $\eta_{k}=\delta_{m_{k}} \cap \sigma_{p_{k}}$ let us set

$$
\phi_{j}=\sum_{k=1}^{j} m_{k} \chi_{\eta_{k}} ; \quad j=1,2, \cdots,
$$

where $\chi_{\eta}$ denotes the characteristic function corresponding to the set $\eta$. It follows immediately that $x_{0} \in D\left(S\left(\phi_{j}\right)\right), j=1,2, \cdots ; 0 \preceq S\left(\phi_{1}\right) x_{0}$ $\preceq S\left(\phi_{2}\right) x_{0} \preceq \ldots$ and

$$
\begin{aligned}
\left|S\left(\phi_{j}\right) x_{0}\right|=\left|\sum_{k=1}^{j} m_{k} P\left(\eta_{k}\right) x_{0}\right| \leqq\left|\sum_{k=1}^{j}\left(m_{k}-1\right) P\left(\eta_{k}\right) x_{0}\right| & \\
& +\left|P\left(\bigcup_{k=1}^{j} \eta_{k}\right) x_{0}\right| .
\end{aligned}
$$

Hence, by Lemma 1 we have

$$
\left|S\left(\phi_{j}\right) x_{0}\right| \leqq\left|\sum_{k=1}^{j} \int_{\eta_{k}} g(\omega) P(d \omega) x_{0}\right|+\left|x_{0}\right|
$$

and since the convergence is uniform on $\bigcup_{k=1}^{j} \eta_{k}$

$$
\left|S\left(\phi_{j}\right) x_{0}\right| \leqq K+\left|x_{0}\right|, \quad j=1,2, \cdots .
$$

Furthermore, $\phi(\omega)=\sup _{j} \phi_{j}(\omega)=m_{k} \geqq g(\omega)$ for $\omega \in \eta_{k}$ which implies in view of Lemma 1, part (b) that $x_{0} \notin D(S(\phi))$. Thus, the sequence $\left\{S\left(\phi_{j}\right) x_{0}\right\}$ has no limit. The following arguments are similar to those used by R. C. James in [5, Lemma 1]. Since the sequence $\left\{S\left(\phi_{j}\right) x_{0}\right\}$ is not convergent one can easily construct an increasing sequence of integers $\left\{j_{n}\right\}$ such that $\left|S\left(\phi_{j_{n+1}}\right) x_{0}-S\left(\phi_{j_{n}}\right) x_{0}\right| \geqq \epsilon ; n=1,2, \cdots$ for some $\epsilon>0$. Set $\psi_{n}=\phi_{j_{n+1}}-\phi_{j_{n}}$ and remark that the functions $\psi_{n}$ have disjoint supports and

$$
\epsilon \leqq\left|S\left(\psi_{n}\right) x_{0}\right| \leqq 2\left(K+\left|x_{0}\right|\right), \quad n=1,2, \cdots
$$

For any sequence $\left(\alpha_{n}\right) \in c_{0}$ observe that

$$
\sum_{n=p}^{q} \alpha_{n} \psi_{n}(\omega) \leqq \max _{p \leq n \leq q}\left|\alpha_{n}\right| \phi_{j_{q+1}}(\omega)
$$


which implies in view of Lemma 1, part (b) that

$$
\left|\sum_{n=p}^{q} \alpha_{n} S\left(\psi_{n}\right) x_{0}\right| \leqq \max _{p \leq n \leq q}\left|\alpha_{n}\right|\left(K+\left|x_{0}\right|\right) .
$$

Consequently, $\sum_{n=1}^{\infty} \alpha_{n} S\left(\psi_{n}\right) x_{0}$ converges and

$$
\left|\sum_{n=1}^{\infty} \alpha_{n} S\left(\psi_{n}\right) x_{0}\right| \leqq\left(K+\left|x_{0}\right|\right) \sup _{n}\left|\alpha_{n}\right| \text {. }
$$

On the other hand, according to Lemma 1, part (c)

$$
\left|\sum_{n=1}^{\infty} \alpha_{n} S\left(\psi_{n}\right) x_{0}\right| \geqq\left|\alpha_{n}\right|\left|S\left(\psi_{n}\right) x_{0}\right| \geqq \epsilon\left|\alpha_{n}\right|, \quad n=1,2, \cdots,
$$

i.e.

$$
\left|\sum_{n=1}^{\infty} \alpha_{n} S\left(\psi_{n}\right) x_{0}\right| \geqq \epsilon \sup _{n}\left|\alpha_{n}\right|
$$

Thus the subspace $\operatorname{clm}\left\{S\left(\psi_{n}\right) x_{0} ; n=1,2, \cdots\right\}$ is isomorphic to $c_{0}$, which contradicts our hypothesis.

Q.E.D.

The next step will be to study $\mathfrak{X}^{*}$, the conjugate of $\mathfrak{X}$. Let $S(f)^{*}$ $=\int f(\omega) P^{*}(d \omega)$ be the adjoint of the closed, densely defined operator $S(f)=\int f(\omega) P(d \omega), D\left(S(f)^{*}\right)$ its domain and $x_{0} * \in\left(\mathfrak{X}^{(r)}\right)^{*}$ the functional already introduced in the proof of the previous lemma whose construction is given by W. G. Bade [2, Theorem 4.3]. According to W. G. Bade [2, Theorem 8.4]

$$
\mathfrak{X}^{*}=\left\{S(f)^{*} x_{0}^{*} \mid x_{0}^{*} \in D\left(S(f)^{*}\right)\right\}
$$

and hence $\left(\mathfrak{X}^{(r)}\right)^{*}=\left\{S(f)^{*} x_{0}^{*} \mid x_{0}^{*} \in D\left(S(f)^{*}\right), f\right.$ real $\}$. One can easily see that $\left(\mathfrak{X}^{(r)}\right)^{*}$ with the order $S\left(f_{1}\right) * x_{0}^{*} \preceq S\left(f_{2}\right) * x_{0}^{*}$ whenever $f_{1}(\omega)$ $\leqq f_{2}(\omega)$ a.e. in $\Omega$ is also a complete Riesz normed space (see also W. A. J. Luxemburg and A. C. Zaanen [8, Note VII, Theorem 22.5]).

Lemma 4. If no subspace of $\mathfrak{X}^{(r)}$ is isomorphic to $l_{1}$ then for every decreasing sequence $S\left(f_{1}\right)^{*} x_{0}^{*} \succeq S^{*}\left(f_{2}\right) x_{0}^{*} \succeq \ldots$ whose greatest lower bound is 0 we have $\lim _{n \rightarrow \infty}\left|S^{*}\left(f_{n}\right) x_{0}^{*}\right|=0$.

Proof. Suppose there exists a decreasing sequence $S\left(h_{n}\right) * x_{0}^{*}$ $\epsilon\left(\mathfrak{X}^{(r)}\right)^{*}$ such that $\lim _{n \rightarrow \infty} h_{n}(\omega)=0$ a.e. in $\Omega$ and $\left|S\left(h_{n}\right)^{*} x_{0}^{*}\right| \geqq \epsilon$ for some $\epsilon>0$. By arguments already used in the proof of the previous lemma we can construct a sequence of Borel sets $\Omega_{\supset} \Omega_{1} \supset \Omega_{2} \supset \ldots$ $\supset \Omega_{p} \supseteq \cdots$ such that $\left\{h_{n}(\omega)\right\}$ converges uniformly for $\omega \in \Omega_{p}{ }^{\prime}=$ $\Omega-\Omega_{p}, p=1,2, \cdots$, and $\cap_{p=1}^{\infty} \Omega_{p}=\varnothing$. Obviously for every $p$ there 
exists an integer $n_{p}$ (and we can assume that $n_{1}<n_{2}<\cdots<n_{p}$ $<\cdots)$ for which $\left|S\left(h_{n_{p}} \chi_{\Omega^{\prime}}{ }^{\prime \prime}\right)^{*} x_{0}^{*}\right|<\epsilon / 2$. Thus

$$
\begin{aligned}
\left|S\left(h_{1} \chi_{\Omega_{p}}\right)^{*} x_{0}^{*}\right| & \geqq\left|S\left(h_{n_{p}} \chi_{\Omega_{p}}\right)^{*} x_{0}^{*}\right| \geqq\left|S\left(h_{n_{p}}\right)^{*} x_{0}^{*}\right|-\left|S\left(h_{n_{p}} \chi_{\Omega^{\prime}}{ }^{\prime \prime}\right)^{*} x_{0}^{*}\right| \\
& \geqq \epsilon-\epsilon / 2=\epsilon / 2, \quad p=1,2, \cdots .
\end{aligned}
$$

Therefore we can find vectors $x_{p}=S\left(g_{p}\right) x_{0} \in \mathfrak{X}^{(r)}$ with $\left|S\left(g_{p}\right) x_{0}\right|=1$ and such that

$$
\left[S\left(h_{1}\right)^{*} x_{0}^{*}\right]\left[S\left(g_{p} \chi_{\Omega_{p}}\right) x_{0}\right] \geqq \epsilon / 4, \quad p=1,2, \cdots .
$$

Consequently $\left[S\left(h_{1}\right)^{*} x_{0}^{*}\right]\left[S\left(\left|g_{p}\right| \chi_{\Omega_{p}}\right) x_{0}\right] \geqq \epsilon / 4, p=1,2, \cdots$.

Since in general the functions $\left|g_{p}\right| \chi_{\Omega_{p}}$ have no disjoint supports one can find an increasing sequence of integers $\left\{p_{s}\right\}$ such that the functions $\phi_{s}=\left|g_{p_{s}}\right| \chi_{\Omega_{p_{s}}-\Omega_{p_{s+1}}} ; s=1,2, \cdots$, have disjoint supports and

$$
\left[S\left(h_{1}\right)^{*} x_{0}^{*}\right]\left[S\left(\phi_{s}\right) x_{0}\right] \geqq \epsilon / 8 ; \quad s=1,2, \cdots .
$$

Hence, for any sequence $\left(\alpha_{\varepsilon}\right) \in l_{1}$ we obtain

$$
\begin{aligned}
\left|S\left(h_{1}\right)^{*} x_{0}^{*}\right| \sum_{s=1}^{\infty}\left|\alpha_{s}\right| & \geqq\left|S\left(h_{1}\right)^{*} x_{0}^{*}\right|\left|\sum_{s=1}^{\infty} \alpha_{s} S\left(\phi_{s}\right) x_{0}\right| \\
& \geqq\left|S\left(h_{1}\right)^{*} x_{0}^{*}\right|\left|S\left(\sum_{s=1}^{\infty} \alpha_{s} \phi_{s}\right) x_{0}\right| \\
& =\left|S\left(h_{1}\right)^{*} x_{0}^{*}\right|\left|S\left(\sum_{s=1}^{\infty}\left|\alpha_{s}\right| \phi_{s}\right) x_{0}\right| \\
& \geqq\left[S\left(h_{1}\right)^{*} x_{0}^{*}\right]\left[S\left(\sum_{s=1}^{\infty}\left|\alpha_{s}\right| \phi_{s}\right) x_{0}\right] \\
& =\sum_{s=1}^{\infty}\left|\alpha_{s}\right|\left[S\left(h_{1}\right)^{*} x_{0}^{*}\right]\left[S\left(\phi_{s}\right) x_{0}\right] \geqq \frac{\epsilon}{8} \sum_{s=1}^{\infty}\left|\alpha_{s}\right|,
\end{aligned}
$$

i.e., $l_{1}$ is isomorphic to the subspace $\operatorname{clm}\left\{S\left(\phi_{s}\right) x_{0} ; s=1,2, \cdots\right\}$, which is a contradiction.

Q.E.D.

TheOREM 5. The cyclic space $\mathfrak{X}=\mathfrak{M}\left(x_{0}\right)$ is reflexive if and only if no subspace of it is isomorphic to either $l_{1}$ or $c_{0}$.

Proof. Since every subspace of a reflexive space is also reflexive no subspace of $\mathfrak{X}$ can be isomorphic to $l_{1}$ or $c_{0}$ provided $\mathfrak{X}$ is reflexive. To prove the converse notice first that it suffices to show that $\mathfrak{X}^{(r)}$ is reflexive. For this purpose we shall use Theorem A. Indeed, condition (a) of this theorem holds in view of a theorem of H. Nakano [11, pp. 
321-322] (see also W. A. J. Luxemburg and A. C. Zaanen [8, Note $\mathrm{X}$, Theorem 33.4]), the Lebesgue Dominated Convergence Theorem for vector measures and the fact that $\mathfrak{X}^{(r)}$ is $\sigma$-Dedekind complete. Condition (b) follows from W. A. J. Luxemburg and A. C. Zaanen [8, Note $\mathrm{X}$, Theorem 33.8], (used for $\left(\mathfrak{X}^{(r)}\right)^{*}$ ), again the Lebesgue Dominated Convergence Theorem, and Lemma 4 provided no subspace of $\mathfrak{X}^{(r)}$ is isomorphic to $l_{1}$. Finally, if no subspace of $\mathfrak{X}^{(r)}$ is isomorphic to $c_{0}$, Lemma 3 and W. A. J. Luxemburg and A. C. Zaanen [8, Note XI, Theorem 34.2] imply that condition (c) is also satisfied. This completes the proof.

Corollary 6. If $\mathfrak{X}^{* *}$ is separable then $\mathfrak{X}$ is reflexive.

Proof. If $\mathfrak{X}$ has a subspace isomorphic to either $c_{0}$ or $l_{1}$ then $\mathfrak{X}^{* *}$ cannot be separable since $\left(c_{0}\right)^{* *}=m$ and $l_{1}^{*}=m$ ( $m$ denotes the space of all bounded sequences) and $m$ is not separable.

REMARKS. 1. This corollary can be proved directly by using Lemma 2 and another result of $T$. Ogasawara [12, Chapter V; $\$ 4$, Theorem 3 ] (see also W. A. J. Luxemburg [9, Theorem 45.1]).

2. Corollary 6 is not true for an arbitrary Banach space (cf. R. C. James [5]).

3. In connection with the proof of Lemma 4, one can observe that if $e_{s}$ denotes the support of $\phi_{s}$ then

$$
P S(f) x_{0}=\sum_{s=1}^{\infty} \frac{\left[S\left(h_{1}\right)^{*} x_{0}^{*}\right]\left[S\left(f \chi_{e s}\right) x_{0}\right]}{\left[S\left(h_{1}\right)^{*} x_{0}^{*}\right]\left[S\left(\phi_{1}\right) x_{0}\right]} S\left(\phi_{s}\right) x_{0}
$$

is a bounded projection (with norm $\leq(8 / \epsilon)\left|S\left(h_{1}\right) * x_{0}^{*}\right|$ ) onto the subspace $\operatorname{clm}\left\{S\left(\phi_{s}\right) x_{0} ; s=1,2, \cdots\right\}$ which is isomorphic to $l_{1}$. Similar arguments show that in Lemma 3 the subspace $\operatorname{clm}\left\{S\left(\phi_{n}\right) x_{0} ; n=1\right.$, $2, \cdots\}$ (which is isomorphic to $c_{0}$ ) is also the range of a projection with norm $\leq\left(K+\left|x_{0}\right|\right) / \epsilon$.

\section{REFERENCES}

1. W. G. Bade, On Boolean algebras of projections and algebras of operators, Trans. Amer. Math. Soc. 80 (1955), 345-359.

2. - A multiplicity theory for Boolean algebras of projections in Banach spaces, Trans. Amer. Math. Soc. 92 (1959), 508-530.

3. N. Dunford, Spectral operators, Pacific J. Math. 4 (1954), 321-354.

4. N. Dunford and J. Schwartz, Linear operators. I, Interscience, New York, 1958.

5. R. C. James, Bases and reflexivity of Banach spaces, Ann. of Math. 52 (1950), 518-527.

6. J. Lindenstrauss and M. Zippin, Banach spaces with a unique unconditional basis, J. Functional Analysis 3 (1969), 115-125. 
7. - Banach spaces with sufficiently many Boolean algebras of projections, J. Math. Anal. Appl. 25 (1969), 309-320.

8. W. A. J. Luxemburg and A. C. Zaanen, Notes on Banach functions spaces. VI, VII, Nederl. Akad. Wetensch. Proc. Ser. A 66 (1963), 665-668, 669-681; X, XI, XIII, ibid. 67 (1964), 495-506, 507-518, 530-543.

9. W. A. J. Luxemburg, Notes on Banach function spaces, $\mathrm{XIV}_{\mathrm{A}}$, Nederl. Akad. Wetensch. Proc. Ser. A 68 (1965), 229-239.

10. C. A. McCarthy and L. Tzafriri, Projections in $\mathscr{L}_{\mathbf{1}}$ and $\mathscr{L}_{\infty}$ spaces, Pacific J. Math. 26 (1968), 529-546.

11. H. Nakano, Semi-ordered linear spaces, Japan Soc. for Promotion of Science, Tokyo, 1955.

12. T. Ogasawara, Vector lattices, Tokyo, 1948. (Japanese)

13. A. Pełczyński, Projections in certain Banach spaces, Studia Math. 19 (1960), 209-228.

14. L. Tzafriri, $A$ characterization of $L_{p}$ and $c_{0}$-spaces, Studia Math. 32 (1969), (to appear).

University of Minnesota 